\title{
Symmetry Invariance of Multiagent Formations in Self-Pursuit
}

\author{
Joshua A. Marshall, Member, IEEE, and Mireille E. Broucke, Member, IEEE
}

\begin{abstract}
Structure in the interconnection topology among individuals of a multiagent system plays a fundamental role in the system's steady-state and transient behaviors. This paper explores how certain interconnection topologies influence symmetry in a multiagent system's trajectories. It is shown how circulant connectivity preserves rotation, and in particular instances, dihedral group symmetries in a formation of locally interacting planar integrators. Moreover, it is revealed to what extent circulant connectivity is also necessary in order that symmetric formations remain symmetric under the multiagent system's dynamics.
\end{abstract}

Index Terms-Cooperative control, multiagent formations, information flow, symmetry groups, circulant matrices.

\section{INTRODUCTION}

"It's a basic principle: Structure always affects function," says Steven Strogatz in his book entitled Sync [1, p. 237]. This paper explores how the interconnection structure among locally interacting agents influences, in particular, the invariance of discrete symmetries in its trajectories. Consider the problem of dynamic target tracking using a team of $n>1$ autonomous mobile robots. This task requires that agents act as a mobile and reconfigurable sensor array. Suppose each agent is equipped with a target-tracking sensor (e.g., an ultrasonic sensor, a laser range finder, or a CCD camera) that, when combined with the sensor readings of other agents, can be utilized by a central observer to estimate the location of a target. If the sensors measure distances to the target, then it can be shown that a configuration that optimizes the estimate is one in which the sensors are uniformly placed in a circular fashion around the target [2]. This optimal sensor placement is "symmetrical," in the sense that the configuration remains optimal under rotations by $2 \pi / n$ about the target. The problem of symmetrical arrangement of agents in a formation has applications in area coverage [3], [4], [5], sampling of distributed data [6], antenna arrays and reconfigurable satellite dishes [7], estimation and localization [8], and in mobile communication networks to reduce power usage and increase the quality of transmissions [9].

The problem of achieving and maintaining symmetry in multiagent formations has been widely studied [10], [11],

Manuscript revised January 31, 2008. This work was supported in part by the Natural Science and Engineering Research Council of Canada (NSERC) and the Walter C. Sumner Foundation.

J. A. Marshall is with the Department of Mechanical and Aerospace Eng., Carleton University, Ottawa, ON, K1S 5B6 Canada (e-mail: marshall@mae.carleton.ca); phone: (613) 520-2600 ext. 3004; This research was carried out while the first author was with the University of Toronto.

M. E. Broucke (corresponding author) is with the Edward S. Rogers Sr. Department of Electrical and Computer Engineering, University of Toronto, Toronto, ON, M5S 3G4 Canada (e-mail: broucke@control.toronto.edu); phone: (416) 978-0803; fax: (416) 978-0804.
[12]. For example, distributed heuristic algorithms for the formation of geometric patterns in the plane (e.g., circles and polygons) are investigated in [13]. Artificial potentials are used to generate stable symmetric formations by inserting virtual leaders among the agents in [14]. A method for stabilizing multiple agents to rigidly constrained formations, while moving along a desired path, is examined in [15]. In [16], a hybrid control strategy is employed to achieve stability for a desired formation, irrespective of its symmetry. Symmetric formations have been considered for a variety of types of agents from satellites [17] to wheeled vehicles [18], [19], [20].

How information flow influences the stability of formations is studied in [21]. Of relevance to the current work is [22], which exploits the symmetry in a network of coupled identical dynamical systems to classify invariant manifolds of the overall system dynamics with respect to their stability. Hence, "stability in the network descends from its topology" [22, p. 67]. Symmetry in the interconnection structure is also exploited in [23], which studies the problem of distributed controller synthesis for large arrays of spatially interconnected systems.

The present research is especially influenced by [24] and [25], wherein a circulant interconnection structure among multiple agents is utilized to deduce the overall steadystate behavior of the agents. In particular, [24] studies the asymptotic behavior of a collection of agents in discrete-time circulant pursuit. Similarly, [25] studies the stability of certain geometric patterns for a collection of continuous-time fixedspeed agents in cyclic pursuit.

This paper studies connectivity as it relates to the problem of choosing distributed controllers that inherently preserve symmetric formations. Designing or studying the stability of symmetric formations, as in [15], [16], [22], [14], [21], is not examined here. We identify interconnection structures that naturally result in invariant manifolds corresponding to formation symmetries. This paper considers $n>1$ identical planar integrators in self-pursuit, each endowed with only relative sensing capabilities, and with a fixed sensing topology. It is revealed how the information flow structure among agents influences symmetry in the multiagent system's trajectories. In particular we show that the only agent interconnection topology that preserves finite symmetry groups in the plane is a circulant interconnection structure. Under the assumption of linear dynamics and relative sensor information, the agents will converge to their center of mass, diverge away from the center of mass, or form a circle. Our concern in this paper is not with the asymptotic behavior of the agents but with the shape of the formation as the dynamics evolve. See [26], [27], [28] for related studies on control of the shape of a formation. 
Our results can be viewed as a first step toward more complex frameworks such as nonlinear agent dynamics, time-varying sensing topologies, multi-agent systems with fixed external references, and control strategies other than linear pursuit.

The motivation for studying this problem is two-fold. One is as a first step toward a more general theoretical framework for symmetry invariance in multi-agent systems. The second is because symmetric arrangements of agents are useful in applications. However, we wish to emphasize that is the first motivation which is more relevant in this paper. We study an idealized problem of rendezvous with an idealized vehicle model of point mass agents, using among the simplest communication structures: self-pursuit. Each of these choices represents an abstraction of real-world situations. Our emphasize is on the theoretical question of what control behaviors can be achieved under a severe restriction on sensor data. In the literature cited above the emphasis has been on achieving rendezvous and consensus alone, whereas the theme of this paper is to show other desirable behaviors can be achieved. Ultimately, a compendium of useful collective behaviors could be classified for a given sensor structure. A next step in the research is to understand how the simplifying assumptions in our framework can be removed while retaining the same set of desirable collective behaviors.

In summary, the contributions of the paper are as follows: first we study cyclic group symmetries in planar multi-agent formations. We define the notion of cyclic formation symmetry and relate this to a permutation applied to the agent indices. We show that this permutation is always similar (in the sense of similar matrices) to a certain power of the fundamental permutation matrix, assuming that a canonical labeling of the agent indices is adopted. The idea of a canonical labeling for cyclic symmetries, which is not new [29], [30], is contrasted with repositioning the agents. Using this labeling, we show that cyclic formation symmetry is invariant under a circulant communcation structure. Invariance is obtained by showing that the agents evolve on a complex linear subspace, and the dynamics on this subspace are explicitly characterized. Next, we explore the extent to which circulant communication structure is necessary for cyclic formation symmetry invariance. By way of an example, we show that a cyclic group symmetry may be invariant even of the communication structure is not circulant. However, if a cyclic group symmetry and all of its subgroups are invariant under the multiagent dynamics, then the communication structure must be circulant. Next, we consider the question of whether all of the above results can be explained simply by studying the topology of the sensor graph. We introduce the notion of graph symmetry - analogous to formation symmetry - and the notion of a structurally circulant matrix - analogous to a circulant matrix - notions which regard only the associated graph structures. It is shown by way of example that graph symmetry is not sufficient to preserve cyclic group symmetries. Finally, we briefly address dihedral symmetries. We define dihedral group symmetry and show, by way of example, that dihedral symmetries are generally not preserved by a circulant communication structure. However, a special class of dihedral symmetries in which the agents lie on a circle do have this property.
The paper is organized as follows. Section II begins with notation and background. Section III introduces systems of agents in self-pursuit and some associated concepts in algebraic graph theory. Next, in Section IV, it is shown how circulant connectivity preserves cyclic group symmetries in multiagent formations. Section V reveals to what extent circulant connectivity is necessary in order that symmetric formations remain invariant under the system's dynamics. Finally, Sections VI and VII discuss graph symmetry and dihedral symmetry invariance, respectively.

\section{NOTATION AND BACKGROUND}

This section introduces some notation and background material relating to permutations and symmetry groups, particularly cyclic and dihedral symmetries.

\section{A. Permutations}

Let $\mathcal{N}:=\{1,2, \ldots, n\}$. A bijection $\sigma: \mathcal{N} \rightarrow \mathcal{N}$, is called a permutation of the set $\mathcal{N}$. In general, one can write $\sigma(1)=i_{1}$, $\sigma(2)=i_{2}, \ldots, \sigma(n)=i_{n}$, or [31, pp. 24-25],

$$
\sigma:\left(\begin{array}{cccc}
1 & 2 & \cdots & n \\
i_{1} & i_{2} & \cdots & i_{n}
\end{array}\right) .
$$

Associated with every permutation $\sigma$ is a square matrix, denoted $P_{\sigma}$, of order $n$. Given an $n \times n$ matrix $A=\left[a_{i k}\right], P_{\sigma}$ is such that $P_{\sigma} A=\left[a_{\sigma(i), k}\right]$ and, therefore, that $P_{\sigma} A P_{\sigma}^{\top}=$ $\left[a_{\sigma(i), \sigma(k)}\right]$ (e.g., $\Pi_{n}$ is the matrix corresponding to $\sigma(i)=$ $i+1)$. Let $\sigma^{l}(i):=\sigma \circ \sigma \circ \cdots \circ \sigma(i)$, the permutation $\sigma$ applied $l$ times to element $i \in \mathcal{N}$. Every $i \in \mathcal{N}$ generates a subset of $\mathcal{N}$ called a cycle of length $l$, where $l$ is the least positive integer such that $\sigma^{l}(i)=i$. In general, a permutation $\sigma$ can be factored into a product of disjoint cycles. This factorization is unique up to the ordering of factors (which are disjoint cycles). A permutation is called primitive if it has only one factor (which has full length $n$ ).

\section{B. Cyclic and Dihedral Group Symmetry}

It is assumed that the reader is familiar with some basic group theory; good references are [29], [32]. In this paper, we utilize two abstract finite groups, the cyclic and dihedral groups. A group is called cyclic when all its elements are powers $g^{k}$ of some one element $g$. For any element $g$ in a group $G$, the set $\left\{g^{k}: k \in \mathbb{Z}\right\}$ defines the cyclic subgroup of $G$ generated by $g$. If $g^{m}=g_{I}$ for some positive integer $m$, then the group generated by $g$ is a finite group. If $m$ is the least positive integer for which this is true, then $m$ is the group's order and the group is $\left\{g_{I}, g, g^{2}, \ldots, g^{m-1}\right\}$. Alternatively, the finite cyclic group of order $m$ has the presentation $\left\langle g: g^{m}=\right.$ $\left.g_{I}\right\rangle$.

An example of the abstract cyclic group in the plane is the rotation symmetry (here called the cyclic group in $\mathbb{R}^{2}$ ), which, roughly speaking, means an object looks the same after a given rotation in the plane.

Definition 1 (Cyclic Group in $\mathbb{R}^{2}$ ): The cyclic group in $\mathbb{R}^{2}$ of order $m$, denoted $C_{m}$, is the finite cyclic group generated by a rotation through $2 \pi / m$ about the origin. 
We say that a subset $\mathcal{U} \subset \mathbb{R}^{2}$ has symmetry $C_{m}$ if the cyclic group $C_{m}$ is a symmetry group of $\mathcal{U}$. Every finite subgroup of $I\left(\mathbb{R}^{2}\right)$, the group of isometries of $\mathbb{R}^{2}$, leaves at least one point invariant [29, p. 44]. In this paper, it is assumed (without loss of generality) that this point is always the origin.

Consider two group elements: $g_{R}$ and $g_{F}$ in $G$ (which can be interpreted as a rotation and reflection in $\mathbb{R}^{2}$, respectively). Then the set $\left\{g_{I}, g_{R}, g_{R}^{2}, \ldots, g_{R}^{m-1}, g_{F}, g_{R} g_{F}, g_{R}^{2} g_{F}, \ldots, g_{R}^{m-1} g_{F}\right\}$ defines the finite dihedral group of order $2 m$. Alternatively, this group has the presentation $\left\langle g_{R}, g_{F}: g_{R}^{m}=g_{F}^{2}=\right.$ $\left.\left(g_{R} g_{F}\right)^{2}=g_{I}\right\rangle$.

Definition 2 (Dihedral Group in $\mathbb{R}^{2}$ ): The dihedral group in $\mathbb{R}^{2}$ of order $2 m$, denoted $D_{m}$, is the finite dihedral group generated by $m$ rotations through $2 \pi / m$ about the origin and $m$ reflections about lines through the origin that make angles of $\pi / m$ with each other.

The dihedral group $D_{m}$ is the complete symmetry group of an $m$-sided regular polygon [29, p. 46]. We say that $\mathcal{U} \subset$ $\mathbb{R}^{2}$ has symmetry $D_{m}$ if the regular polygon group $D_{m}$ is a symmetry group of $\mathcal{U}$. Note that $C_{m}$ is a subgroup of $D_{m}$; i.e., symmetry $D_{m}$ implies symmetry $C_{m}$, but not the converse.

We are interested only in finite subgroups of the isometry group $I\left(\mathbb{R}^{2}\right)$, since a real formation of agents (or subset $\mathcal{U} \subset \mathbb{R}^{2}$ ), not all collocated, can have only a finite set of symmetries. Given some positive integer $m, C_{m}$ and $D_{m}$ are the only finite subgroups of $I\left(\mathbb{R}^{2}\right)$ [33]. Thus, cyclic and dihedral group symmetries in $\mathbb{R}^{2}$ are the only groups we study.

\section{Agents in Pursuit}

Agents are viewed as points in the complex plane, denoted C. Consider $n>1$ planar agents, $z_{1}(t), z_{2}(t), \ldots, z_{n}(t) \in$ $\mathbb{C}$, evolving in time $t$. Suppose that each agent is a simple integrator; i.e., $\dot{z}_{i}(t)=u_{i}(t) \in \mathbb{C}, i=1,2, \ldots, n$, where $u_{i}(t)$ is the control input. Assume that these kinematic agents have only relative sensing capabilities (i.e., the agents do not have information about their own or other agents states in terms of an absolute coordinate system) and, therefore, that the inputs $u_{i}(t)$ are of the type

$$
u_{i}(t)=\sum_{k \neq i}^{n} a_{i k}\left(z_{k}(t)-z_{i}(t)\right), i=1,2, \ldots, n .
$$

That is, the aggregate multiagent system is of the form

$$
\dot{z}(t)=A z(t)
$$

where $z(t)=\left(z_{1}(t), \ldots, z_{n}(t)\right) \in \mathbb{C}^{n}$ and $A$ is a weighted adjacency matrix describing the flow of information between agents. A direct consequence of the relative sensing limitation is the property that follows.

Property 1: The adjacency matrix $A$ has zero row-sums (i.e., $A\left[\begin{array}{llll}1 & 1 \ldots 1\end{array}\right]^{\top}=0$ ).

Hence, if the agents are all collocated, then there is no motion.

For the system of $n>1$ agents, the information flow between agents is directed, so this flow of information can be represented by a digraph, denoted $\Gamma=(\mathcal{V}, \mathcal{E})$ [34]. The digraph $\Gamma$ consists of a finite set $\mathcal{V}$ of $|\mathcal{V}|=n$ enumerated vertices, one for each agent, along with a set $\mathcal{E}$ of $|\mathcal{E}| \geq 0$ directed edges $e_{i k}=(i, k) \in \mathcal{E}$, where $i, k \in \mathcal{V}$. The existence of an edge $e_{i k}$ indicates that the $i$-th agent receives information about the $k$-th agent. The adjacenty matrix $A$ associates a weight to each edge of the digraph: the $(i, k)$ element of $A$ is the weight $a_{i k}$ associated with the edge $e_{i k} \in \mathcal{E}$, and is otherwise zero. Alternatively, given an adjacency matrix $A$, one can define an associated digraph, denoted $\Gamma(A)$. If $a_{i k} \neq 0$, then there exists a directed edge in $\Gamma(A)$ from vertex $i$ to $k$.

Information about the formation of agents together with their interconnection topology can be combined into one graph. At each instant $t$, we define a set of locations $\mathcal{V}_{t}=$ $\left\{z_{1}(t), z_{2}(t), \ldots, z_{n}(t)\right\}$ and a set $\mathcal{E}_{t}$ of edge vectors $e_{i k}(t)$ : $\mathcal{V}_{t} \times \mathcal{V}_{t} \rightarrow \mathbb{C}$ such that an edge $e_{i k}(t):=z_{k}(t)-z_{i}(t)$ exists in $\mathcal{E}_{t}$ only if there exists a corresponding edge in $\mathcal{E}$. We refer to the pair $\left(\mathcal{V}_{t}, \mathcal{E}_{t}\right)=: \Gamma(A, z(t))$ as the formation graph (or just graph for short). Fig. 1 provides three example formation graphs.

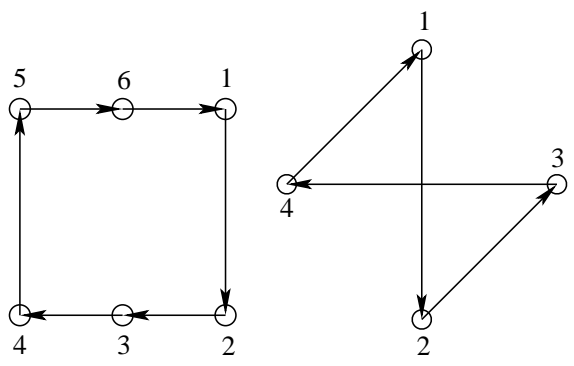

(a)

(b)

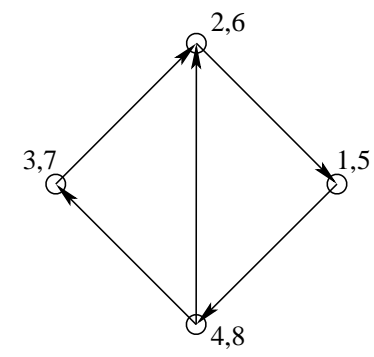

(c)

Fig. 1. Example formation graphs $\Gamma(A, z(t))$.

This paper addresses the following question: What fixed interconnection topologies $\Gamma(A)$ and associated interconnection weights $A=\left[a_{i k}\right]$ preserve rotation and dihedral group symmetries in multiagent formations $z(t) \in \mathbb{C}^{n}$ for all $t \geq 0$ ?

\section{A. Circulant Interconnections}

It will be shown in Sections IV and V that of fundamental significance to the topic of symmetry is a particular interconnection structure in the sensing topology: namely, circulant connectivity. By circulant connectivity it is meant that the adjacency or system matrix $A$ is a circulant matrix [31]; i.e., 
of the form

$$
A=\left[\begin{array}{cccc}
a_{0} & a_{1} & \cdots & a_{n-1} \\
a_{n-1} & a_{0} & \cdots & a_{n-2} \\
\vdots & \vdots & & \vdots \\
a_{1} & a_{2} & \cdots & a_{0}
\end{array}\right]=: \operatorname{circ}\left(a_{0}, a_{1}, \ldots, a_{n-1}\right)
$$

Each row is merely the row above, shifted one element to the right (modulo $n$ ). The matrix is entirely determined by its first row. Let $\kappa \geq 0$ denote the cardinality of the set $\left\{i \in\{1, \ldots, n-1\} \mid a_{i} \neq 0\right\}$; i.e., $\kappa$ represents the degree of coupling between vertices of the circulant digraph $\Gamma(A)$. Denote the fundamental permutation matrix of order $n, \Pi_{n}=$ $\operatorname{circ}(0,1,0, \ldots, 0)$. If there exists a relabeling of the agent indices such that $A$ is subsequently circulant, then the system also has circulant connectivity. Further details about relabeling are provided in Section IV-B.

Following a standard notion, e.g., as in [35], a matrix $A_{1}$ is said to have the same structure as another matrix $A_{2}$, of the same dimensions, if for every zero entry of $A_{1}$ the corresponding entry in $A_{2}$ is also zero, and vice versa. Accordingly, if a square matrix $A$ is such that there exists a circulant matrix $A_{c}$ of the same order and structure as $A$, then $A$ is called structurally circulant. Clearly, the topology of $\Gamma(A)$ is identical to that of $\Gamma\left(A_{c}\right)$. In particular a graph may have a circulant connectivity because the adjacency matrix is structurally circulant, even if it is not circulant. For example, the graphs in Fig. 1a and Fig. 1b correspond to structurally circulant adjacency matrices. This raises a question to be addressed in the sequel: is it sufficient for the adjacency matrix to be circulant, or structurally circulant, to preserve formation symmetries?

If the degree of coupling between individuals is $\kappa=1$ and the off-diagonal element of a circulant matrix $A$ is positive, then (2) becomes the well-known cyclic pursuit (a.k.a., dogs, mice, bugs, or beetles) problem; e.g., agent $i$ "pursues" agent $i+1$, modulo $n$ [36]. Cyclic pursuit has a long and interesting history in the mathematics and physics literature, and the interested reader is referred to [37], [36] for historical accounts. Finally, if the degree of coupling $\kappa=n-1$, then this corresponds to what is often referred to as "all-toall" coupling, since every agent can sense every other agent. Although not every all-to-all coupled matrix $A$ is circulant, every all-to-all coupled $A$ is structurally circulant.

\section{Symmetric Formations AND InVARIANCE}

If we consider only the agent positions and ignore the interagent connections, the configuration of points $z(t) \in \mathbb{C}^{n}$ at time $t$ is referred to as a multiagent formation. The focus of this section is on cyclic group formation symmetries, and the principal result is Theorem 2, which states that if a system has circulant connectivity (see Section III-A), then symmetric formations remain symmetric. Dihedral symmetry is examined in Section VII.

\section{A. Cyclic Group Formations}

Definition 3 ( $C_{m}$ Formation Symmetry): The formation $z(t) \in \mathbb{C}^{n}$ at time $t$ is said to have symmetry $C_{m}$ if there exists a permutation $\sigma: \mathcal{N} \rightarrow \mathcal{N}$ such that

$$
e^{j 2 \pi / m} z(t)=P_{\sigma} z(t) .
$$

That is, by rotating the agents $z(t) \in \mathbb{C}$ through angle $2 \pi / m$ one obtains the same set of points in $\mathbb{C}$, but (generally) with a different labeling. Henceforth, we will simply say that a formation $z(t) \in \mathbb{C}^{n}$ has symmetry $C_{m}$ "with $P_{\sigma}$ " if the vector $z(t)$ satisfies Definition 3 with associated permutation matrix $P_{\sigma}$. Following Definition 3, several remarks are in order. Let $n_{0} \geq 0$ denote the number of agents located at the origin.

Remark 1: If at time $t$ a formation $z(t)$ with $n_{0}=0$ has symmetry $C_{m}$, then $m$ divides $n$. This is because, if one applies the associated constraint (3) in sequence starting from $z_{i}(t) \neq 0$, one obtains at the $n$-th iteration $z_{\sigma^{n}(i)}(t)=$ $e^{j 2 \pi n / m} z_{i}(t)=z_{i}(t)$. Hence, $m=n$ or $m<n$ is a factor of $n$, implying that the cyclic group $C_{m}$ is subgroup of $C_{n}$.

Remark 2: If a formation $z(t)$ with $n_{0}=0$ has symmetry $C_{n}$, then the associated permutation $\sigma$ is primitive. For if not (i.e., $\sigma$ has a cycle of length $l<n$ ), then one obtains at the $l$-th iteration (as in Remark 1) $e^{j 2 \pi l / n} z(t)=P_{\sigma}^{l} z(t)=z(t)$, which can only be true for $l<n$ if $z(t) \equiv 0$.

Remark 3: Suppose a formation $z(t)$ with $n_{0}=0$ has symmetry $C_{m}$, where $m<n$. If there are collocated agents, then it is possible that there exists more than one permutation $\sigma$ such that (3) is satisfied. For instance, the $n=8$ agents in Fig. 1c have symmetry $C_{4}$ with the primitive permutation

$$
\sigma:\left(\begin{array}{ccccc}
1 & 2 & \cdots & 7 & 8 \\
2 & 3 & \cdots & 8 & 1
\end{array}\right)
$$

or, equivalently, $\sigma=(1,2, \ldots, 8)$. However, the constraint (3) also holds with

$$
\sigma:\left(\begin{array}{llllllll}
1 & 2 & 3 & 4 & 5 & 6 & 7 & 8 \\
2 & 3 & 4 & 1 & 6 & 7 & 8 & 5
\end{array}\right)
$$

which has two factors and can be denoted $\sigma=$ $(1,2,3,4)(5,6,7,8)$. Following Remark 1 , it is clear from the geometry of symmetry $C_{m}$ that any factors of $\sigma$ must have a length that is a multiple of $m$.

Consequent to Remark 3, it is assumed in this paper that if a formation $z(t)$ has symmetry $C_{m}$ according to Definition 3 and $n_{0}=0$, then its associated permutation $\sigma$ is one that factors into exactly $n / m$ cycles of length $m$. Let $\operatorname{gcd}(n, q)$ denote the greatest common divisor of the integers $n$ and $q$. The following is a useful fact.

Remark 4: If $m$ divides $n$, then there always exists an integer $q \in\{1,2, \ldots, n-1\}$ such that $\operatorname{gcd}(n, q)=n / m$ since one can always choose $q=n / m$.

\section{B. Canonical Labeling}

Before discussing symmetry invariance, this section establishes a connection between formation symmetry $C_{m}$ and a canonical labeling of the agents. It is shown that agents satisfying the formation symmetry constraint (3) can always be relabeled such that (3) holds with $P_{\sigma}=\Pi_{n}^{q}$, for an appropriate choice of $q$. Note that the permutation corresponding to $P_{\sigma}=$ $\Pi_{n}^{q}$ is $\sigma(i)=i+q, i=1,2, \ldots, n$. This choice of labeling is not new; indeed, some textbooks assume it from the outset when discussing cyclic group symmetry; e.g., [29], [30]. 
Theorem 1: Consider a formation $\tilde{z}(t)$ that has no agents at the origin. Suppose that $\tilde{z}(t)$ has symmetry $C_{m}$ at time $t$ and let $q \in\{1,2, \ldots, n-1\}$ satisfy $\operatorname{gcd}(n, q)=n / m$ (cf. Remark 4). Then, there exists a permutation $\tau$ of the agent locations $z(t)=P_{\tau} \tilde{z}(t)$ such that (3) holds with $P_{\sigma}=\Pi_{n}^{q}$.

Example 1: Consider the formation of agents in Fig. 2a, which has symmetry $C_{5}$ since the constraint (3) holds with $m=5$ and

$$
P_{\sigma}=\left[\begin{array}{llllllllll}
0 & 0 & 0 & 0 & 0 & 1 & 0 & 0 & 0 & 0 \\
0 & 0 & 0 & 0 & 0 & 0 & 0 & 0 & 0 & 1 \\
0 & 1 & 0 & 0 & 0 & 0 & 0 & 0 & 0 & 0 \\
0 & 0 & 1 & 0 & 0 & 0 & 0 & 0 & 0 & 0 \\
0 & 0 & 0 & 1 & 0 & 0 & 0 & 0 & 0 & 0 \\
0 & 0 & 0 & 0 & 0 & 0 & 1 & 0 & 0 & 0 \\
0 & 0 & 0 & 0 & 0 & 0 & 0 & 1 & 0 & 0 \\
0 & 0 & 0 & 0 & 0 & 0 & 0 & 0 & 1 & 0 \\
1 & 0 & 0 & 0 & 0 & 0 & 0 & 0 & 0 & 0 \\
0 & 0 & 0 & 0 & 1 & 0 & 0 & 0 & 0 & 0
\end{array}\right] .
$$

In Fig. 2, the angle between adjacent dotted lines of equal length is $2 \pi / 5$.

Let $q=4$, which satisfies $\operatorname{gcd}(10,4)=10 / 5=2$. By Theorem 1, there exists a permutation $\tau$ of the agent locations such that (3) holds with the permutation matrix $\Pi_{10}^{4}$, as in Fig. 2b. After this repositioning $\tau$, the new permutation $\tilde{\sigma}(i)=i+4$ factors as $\tilde{\sigma}=(1,5,9,3,7)(2,6,10,4,8)$.

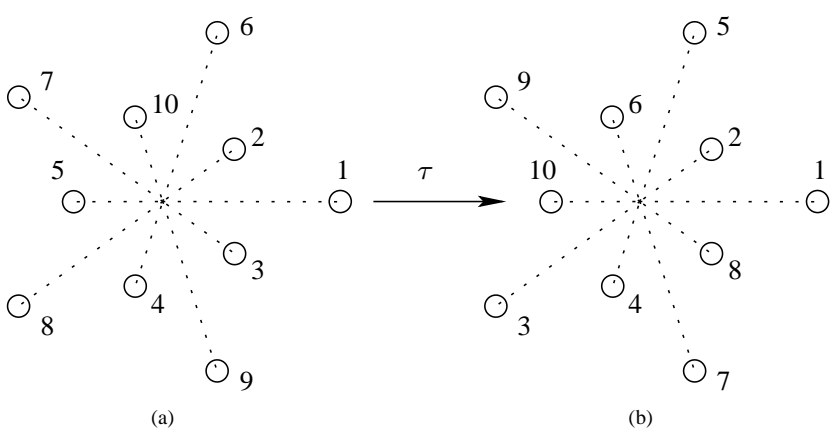

Fig. 2. A formation of $n=10$ agents with $C_{5}$ symmetry.

Let $\tau$ denote the permutation of Theorem 1 that relabels the agents. Notice that by substituting $\tilde{z}(t)=P_{\tau}^{\top} z(t)$ into (3), one obtains

$$
e^{j 2 \pi / m} z(t)=P_{\tau} P_{\sigma} P_{\tau}^{\top} z(t)=\Pi_{n}^{q} z(t) .
$$

Remark 5: If a formation $z(t)$ has symmetry $C_{m}$, then any permutation that relabels the agent indices does not change the symmetry; it changes only the permutation $\sigma$ with which (3) holds. However, repositioning the agents (generally) constitutes a change in the initial formation. For example, swapping the locations of agents 2 and 3 in Fig. 1b will change the group's transient behavior. Instead, if one simultaneously permutes the rows and columns of $A$ by $P_{\tau} A P_{\tau}^{\top}$, one can now view this change as merely a transformation of coordinates given by $P_{\tau}$ or, equivalently, simply a relabeling of the agents.

Henceforth, we will simply say that a formation $z(t) \in \mathbb{C}^{n}$ has symmetry $C_{m}$ "with $P_{\sigma}=\Pi_{n}^{q}$ " if the vector $z(t)$ satisfies Definition 3 with $P_{\sigma}=\Pi_{n}^{q}$ for some $q \in\{1,2, \ldots, n-1\}$ satisfying $\operatorname{gcd}(n, q)=n / m$.
Finally, agents at the origin play no role in symmetry; they merely complicate the labeling. Hence, for the sake of simplicity, it is assumed that $n_{0}=0$ throughout the remainder of this paper.

\section{Symmetry Invariance}

The focus of this paper is on identifying certain interconnection structures that inherently result in invariance of formation symmetries. Following Section IV-B, this naturally leads to the next definition.

Definition $4\left(C_{m}\right.$ Formation Symmetry Invariance): Let $m$ be a divisor of $n$. Formation symmetry $C_{m}$ is said to be invariant under the dynamics (2) if for every $q \in\{1,2, \ldots, n-$ 1) such that $\operatorname{gcd}(n, q)=n / m$ and for every initial formation $z(0) \in \mathbb{C}^{n}$ having symmetry $C_{m}$ with $P_{\sigma}=\Pi_{n}^{q}$, the formation $z(t)$ has formation symmetry $C_{m}$ with $P_{\sigma}=\Pi_{n}^{q}$ for all $t \geq 0$.

The next result shows that given $n$ properly labeled agents, every possible cyclic group symmetry of a formation is invariant when the dynamics are circulant.

Theorem 2: If $A$ is a circulant matrix, then formation symmetry $C_{m}$ is invariant under the dynamics (2) for every $m$ that divides $n$.

Proof: For every $m$ that divides $n$, associated with the constraint (3) at time $t=0$ is a complex linear subspace $\mathcal{M}=\left\{z \in \mathbb{C}^{n}: M z=0\right\} \subset \mathbb{C}^{n}$, where $M=\Pi_{n}^{q}-e^{j 2 \pi / m} I_{n}$. Since $A$ is a circulant matrix, it commutes with $\Pi_{n}[31$, p. 68]. Thus,

$M A=\left(\Pi_{n}^{q}-e^{j 2 \pi / m} I_{n}\right) A=A\left(\Pi^{q}-e^{j 2 \pi / m} I_{n}\right)=A M$.

This implies $\mathcal{M}$ is invariant under the dynamics (2), implying the constraint (3) holds with $P_{\sigma}=\Pi_{n}^{q}$ for all $t \geq 0$.

Example 2: Consider the $n=8$ agents depicted in Fig. 3a. This formation $z(0)$ has symmetry $C_{4}$ with associated permutation $\sigma=(1,3,5,7)(2,4,6,8)$. Let

$$
A=\operatorname{circ}(-1,-1,0,0,0,0,2,0)
$$

be the corresponding multiagent system matrix. Thus, every agent $i \in \mathcal{V}$ is repelled from agent $i+1$, but doubly attracted to agent $i+6$. Fig. $2 \mathrm{~b}$ shows the evolution of the formation starting at $z(0)$ under the dynamics (2) with (4). The fact that the agents converge to the origin is not of interest here. Rather, the dashed and dotted lines connecting the agents $\{1,3,5,7\}$ and $\{2,4,6,8\}$, respectively, form a square at regular intervals during the simulation, highlighting that $C_{4}$ symmetry is preserved.

Corollary 1: Given a permutation $\sigma$, let $\tau$ be such that $P_{\tau} P_{\sigma} P_{\tau}^{\top}=\Pi_{n}^{q}$ (cf. Theorem 1). Let $m$ be any divisor of $n$ and suppose $z(0) \in \mathbb{C}^{n}$ has symmetry $C_{m}$ with permutation matrix $P_{\sigma}$. If $P_{\tau} A P_{\tau}^{\top}$ is a circulant matrix, then the formation $z(t)$ has symmetry $C_{m}$ with $P_{\sigma}$ for all $t \geq 0$.

Proof: If $z(0)$ has symmetry $C_{m}$ with $P_{\sigma}$, Theorem 1 says that there always exists a relabeling of the agents $\tilde{z}(0)=$ $P_{\tau} z(0)$ such that

$$
e^{j 2 \pi / m} \tilde{z}(0)=\Pi_{n}^{q} \tilde{z}(0),
$$

where $q \in\{1,2, \ldots, n-1\}$ satisfies $\operatorname{gcd}(n, q)=n / m$. Viewing $\tilde{z}(t)=P_{\tau} z(t)$ as a transformation of coordinates 


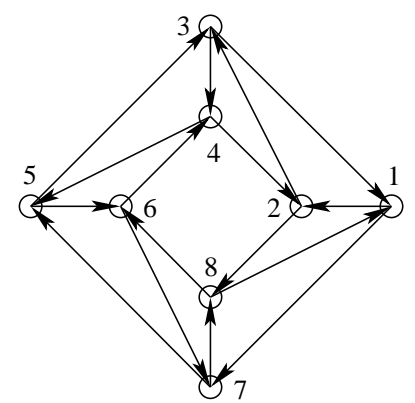

(a) Initial graph $\Gamma(A, z(0))$.

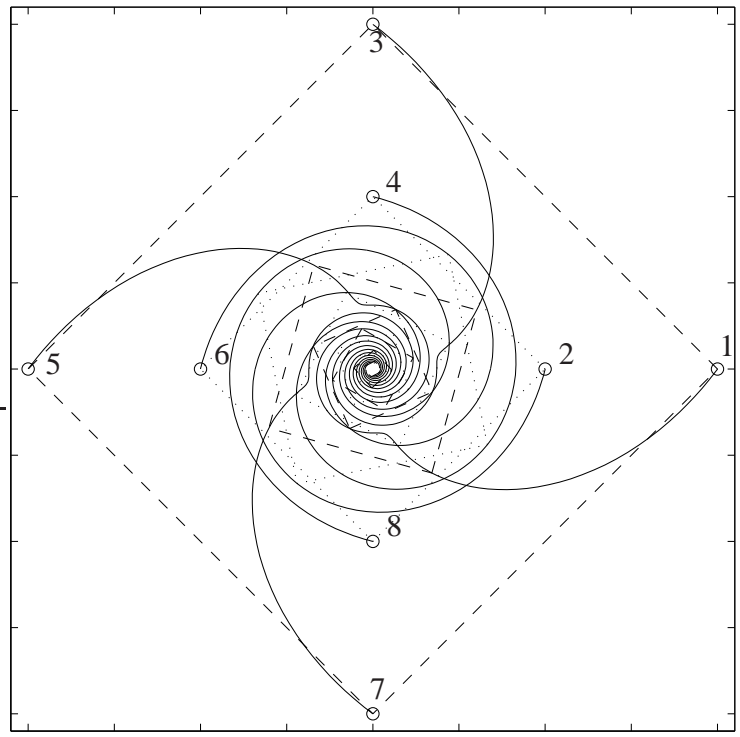

(b) Simulation demonstrating symmetry invariance.

Fig. 3. Initial formation graph and simulation results for Example 2.

implies that (2) becomes

$$
\dot{\tilde{z}}(t)=P_{\tau} A P_{\tau}^{\top} \tilde{z}(t)
$$

If the new system matrix $P_{\tau} A P_{\tau}^{\top}$ is circulant, then (5) and (6) satisfy the conditions of Theorem 2. Therefore, the formation $\tilde{z}(t)$ has symmetry $C_{m}$ with permutation $\Pi_{n}^{q}$ for all $t \geq 0$. If $\tilde{z}(t)$ has symmetry $C_{m}$ for all $t \geq 0$, then $z(t)=P_{\tau}^{\top} \tilde{z}(t)$ also has symmetry $C_{m}$ (with $P_{\sigma}$ ) for all $t \geq 0$. This is because the change of coordinates given by $\tau$ is merely a permutation of the agent locations, which does not alter the symmetry of the formation (cf. Remark 5).

The following example illustrates the previous corollary.

Example 3: Consider a system (2) of $n=4$ agents with

$$
A=\left[\begin{array}{rrrr}
-2 & 2 & 1 & -1 \\
2 & -2 & -1 & 1 \\
-1 & 1 & -2 & 2 \\
1 & -1 & 2 & -2
\end{array}\right]
$$

which is not circulant. Suppose the graph $\Gamma(A, z(0))$ is the same as in Fig. 4a, but with agents 2 and 3 having swapped positions. Hence, the relabeling that takes $P_{\sigma}$ into the form $\Pi_{n}$ is given by

$$
P_{\tau}=\left[\begin{array}{llll}
1 & 0 & 0 & 0 \\
0 & 0 & 1 & 0 \\
0 & 1 & 0 & 0 \\
0 & 0 & 0 & 1
\end{array}\right] .
$$

Moreover, the relabeling yields $P_{\tau} A P_{\tau}^{\top}=\operatorname{circ}(-2,1,2,-1)$. Therefore, following Corollary 1 , the formation $z(t)$ has symmetry $C_{4}$ for all $t \geq 0$.

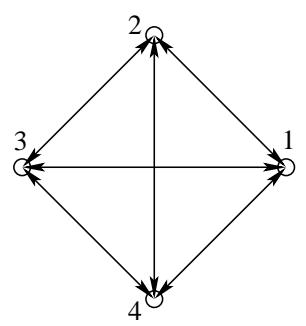

(a)

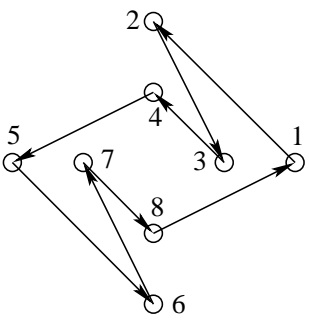

(b)
Fig. 4. Circulant formation graphs $\Gamma(A, z(t))$.

In conclusion, if the multiagent system (2) has an underlying circulant structure (possibly after a change of coordinates; a.k.a., a relabeling), then every cyclic group symmetry, of any initial formation, is invariant under the system's dynamics.

\section{Decomposition of the Dynamics}

Before moving on to necessity, this section briefly explores the complex linear subspace that corresponds to formation symmetry $C_{m}$ in the proof of Theorem 2 .

It was shown in the previous section that, given a canonical labeling, circulant systems preserve cyclic group symmetries (Theorem 2). The complex linear subspace $\mathcal{M}=\{z \in$ $\left.\mathbb{C}^{n}: M z=0\right\} \subset \mathbb{C}^{n}$, where $M=\Pi_{n}^{q}-e^{j 2 \pi / m} I_{n}$ and which characterizes cyclic group symmetries, corresponds to $n-n / m$ independent complex constraints on the motion of the multiagent system (2). In other words, $\operatorname{dimKer} M=n / m$. One way to see this is graphically. For example, consider the $n=10$ agents with symmetry $C_{5}$ in Fig. 2 b. The associated permutation factors into two disjoint cycles; namely, $\sigma=$ $(1,5,8,3,7)(2,6,10,4,8)$. By selecting only two agents, one from each cycle, one can determine the locations of all the remaining agents by performing rotations through $2 \pi / 5$. More generally, one can always write out the cycles generated by a given $C_{m}$ formation of agents $1,2, \ldots, n / m$. These cycles are the disjoint factors of $\sigma(i)=i+q$, where $\operatorname{gcd}(n, q)=n / m$. Since we are allowed to independently specify the locations of the first $n / m$ agents, we have exactly $n / m$ complex degrees of freedom. Hence, there exist $n-n / m$ independent complex constraints on the system.

Let $p:=n / m$ and define $w(t):=\left(z_{1}(t), z_{2}(t), \ldots, z_{p}(t)\right)$. For every $z(t) \in \mathcal{M}, z(t)$ can be written as

$$
z(t)=\left[\begin{array}{llll}
w^{\top}(t) & e^{j 2 \pi / m} w^{\top}(t) & \cdots & \left(e^{j 2 \pi / m}\right)^{m-1} w^{\top}(t)
\end{array}\right]^{\top} .
$$


Observe that if the system matrix $A$ is circulant and of order $n=p \cdot m$, it can be partitioned into precisely $m^{2}$ blocks, each of order $p$. This partitioning causes $A$ to become a block circulant matrix, denoted $A=\operatorname{circ}\left(A_{0}, A_{1}, \ldots, A_{m-1}\right)$, where the blocks $A_{0}, A_{1}, \ldots, A_{m-1}$ are of order $p$; see [31, Section 5.6]. This partitioning allows one to write the $n / m$ dimensional dynamics on $\mathcal{M}$ as

$$
\begin{aligned}
\dot{w}(t) & =\left[\begin{array}{llll}
A_{0} & A_{1} & \cdots & A_{m-1}
\end{array}\right] z(t) \\
& =\sum_{i=0}^{m-1}\left(e^{j 2 \pi / m}\right)^{i} A_{i} w(t) .
\end{aligned}
$$

Example 4: Consider the special case of cyclic pursuit (see Section III-A), with $A=\operatorname{circ}(-1,1,0, \ldots, 0)$. Suppose $z(t) \in$ $\mathbb{C}^{n}$ has symmetry $C_{n}$ with $P_{\sigma}=\Pi_{n}$. Hence, the dimension of the complex dynamics on $\mathcal{M}$ is simply 1 (i.e., there are $n-1$ complex constraints). Let $w(t)=z_{1}(t)$, yielding the dynamics on $\mathcal{M}$,

$$
\dot{w}(t)=\left(e^{j 2 \pi / n}-1\right) w(t) .
$$

Next, consider the agents in Fig. 1a. The formation $z(t)$ of $n=6$ agents has symmetry $C_{2}$ with $P_{\sigma}=\Pi_{n}^{3}$. Suppose the agents are in cyclic pursuit with $A=\operatorname{circ}(-1,1,0, \ldots, 0)$, which is consistent with the graph $\Gamma(A, z(t))$ in the figure. In this case, $w(t)=\left(z_{1}(t), z_{2}(t), z_{3}(t)\right)$. The dynamics on $\mathcal{M}$ have dimension $6 / 2=3$, and are given by

$$
\dot{w}(t)=\left[\begin{array}{rrr}
-1 & 1 & 0 \\
0 & -1 & 1 \\
-1 & 0 & 1
\end{array}\right] w(t)
$$

Using (8), the $3 \times 3$ matrix in the above equation is $A_{0}-A_{1}$, where $A_{0}$ is the upper-left $3 \times 3$ block of $A$ and $A_{1}$ is the upper-right $3 \times 3$ block of $A$.

\section{Circulant Necessity}

Thus far, it has been proved that circulant multiagent systems preserve cyclic group symmetries. The question that is addressed in this section is: To what extent is circulant connectivity also necessary? It is revealed in Theorem 3 , which follows, that circulant connectivity is necessary if symmetry $C_{m}$ is to be invariant under the multiagent system's dynamics for every $m$ that divides $n$.

\section{A. Counterexample}

For any single $m$ dividing $n$, the condition of Theorem 2 that $A$ be circulant is not, in general, necessary for symmetry invariance. The following example illustrates this fact.

Example 5: Consider a system (2) of $n=4$ agents, where the inputs (1) are chosen such that the corresponding system matrix $A$ is the non-circulant matrix

$$
A=\left[\begin{array}{rrrr}
-1 & 1 & 0 & 0 \\
-1 & 0 & 0 & 1 \\
0 & 0 & -1 & 1 \\
1 & 0 & 0 & -1
\end{array}\right]
$$

Consider the initial formation $z(0)$ and graph $\Gamma(A, z(0))$ given in Fig. 5. The formation $z(0)$ has symmetry $C_{4}$ with $P_{\sigma}=\Pi_{4}$.
It can be verified by simulation that, under the dynamics (2) with (10), the formation $z(t)$ has symmetry $C_{4}$ for all $t \geq 0$. However, it can also be verified that there exists an initial formation having symmetry $C_{2}$ (a subgroup of $C_{4}$ ) with $P_{\sigma}=$ $\Pi_{4}^{2}$ such that symmetry $C_{2}$ is not preserved for all $t \geq 0$. In particular, if we select the initial condition $z_{1}(0)=z_{3}(0)$ and $z_{2}(0)=z_{4}(0)$, then the agents remain colinear for all $t$, but for sufficient small $t>0$, agents 1 and 3 pursue 2 and 4 , while agent 2 moves away from 1 and 3 and agent 4 moves toward 2 and 3 . Thus, $C_{2}$ symmetry is instantaneously broken after $t=0$.

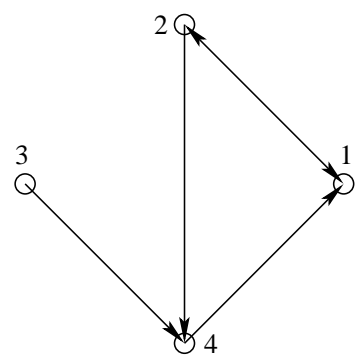

Fig. 5. Non-circulant figure $\Gamma(A, z(0))$ for Example 5

\section{B. A Special Class of Formations}

In studying the necessity of circulant connectivity, it is helpful to employ a special class of formations $z(t)$; namely, those given by the constraint

$$
\omega^{q} z(t)=\Pi_{n} z(t)
$$

for some $q \in\{1,2, \ldots, n-1\}$ and where $\omega:=e^{j 2 \pi / n}$. Notice that the locations $z_{i}(t), i=1,2, \ldots, n$, generated by the constraint (11) all have the same magnitude, and hence lie on a common circle. The following lemma associates a formation satisfying (11) with its symmetry.

Lemma 1: Suppose $\omega^{q} z(t)=\Pi_{n} z(t)$ holds for some $q \in$ $\{1,2, \ldots, n-1\}$ and $z(t) \in \mathbb{C}^{n}$. Then, the formation $z(t)$ has symmetry $C_{m}$, where $m=n / \operatorname{gcd}(n, q)$.

Proof: Let $p:=\operatorname{gcd}(n, q)$ and define $m:=n / p$ and $k_{q}:=q / p$. To show that the formation has symmetry $C_{m}$, we must show there exists a permutation matrix $P_{\sigma}$ such that (3) holds. From (11),

$$
\left(e^{j 2 \pi / n}\right)^{q} z(t)=\left(e^{j 2 \pi / m}\right)^{k_{q}} z(t)=\Pi_{n} z(t) .
$$

By Bézout's identity ${ }^{1}$, there exist integers $l_{q}$ and $l_{m}$ such that $1=\operatorname{gcd}\left(k_{q}, m\right)=l_{q} k_{q}+l_{m} m$. This fact together with (12) yields

$$
e^{j 2 \pi / m} z(t)=\left(e^{j 2 \pi / m}\right)^{l_{q} k_{q}} z(t)=\Pi_{n}^{l_{q}} z(t) .
$$

By letting $P_{\sigma}=\Pi_{n}^{l_{q}}$, one obtains the desired result.

Notice that the proof of Lemma 1 also reveals how formations satisfying the special constraint (11) have symmetry $C_{m}$

${ }^{1}$ Given two nonzero integers $a$ and $b$, Bézout's identity says that there exist integers $c$ and $d$ such that $\operatorname{gcd}(a, b)=a c+b d[38$, Section 1.2, Theorem 1.7]. 
with the canonical labeling introduced in Section IV-B; i.e., (3) holds with $P_{\sigma}=\prod_{n}^{l_{q}}$.

Example 6: Consider the example graphs $\Gamma(A, z(0))$ with $\omega^{q} z(0)=\Pi_{n} z(0)$ given in Fig. 6, where $n=6$. In Fig. $6 \mathrm{a}, q=1$ and the formation has symmetry $C_{6}$ since $m=$ $6 / \operatorname{gcd}(6,1)=6 / 1=6$. In Fig. $6 \mathrm{~b}, q=2$ and the formation has symmetry $C_{3}$ since $m=6 / \operatorname{gcd}(6,2)=6 / 2=3$.

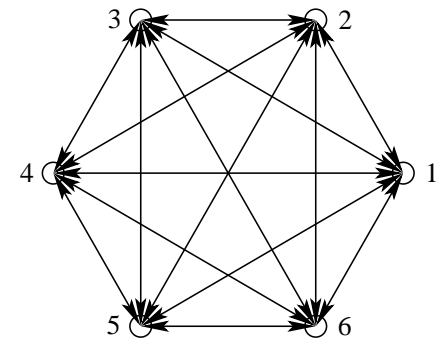

(a) $q=1$

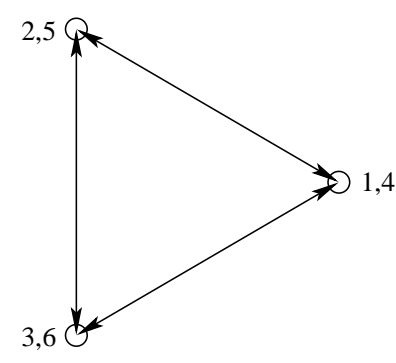

(b) $q=2$
Fig. 6. Example graphs $\Gamma(A, z(0))$ with $\omega^{q} z(0)=\Pi_{6} z(0)$.

Let $v_{q}:=\left(1, \omega^{q}, \omega^{2 q}, \ldots, \omega^{(n-1) q}\right)$, which happens to be the $(q+1)$-th column of $\sqrt{n} F_{n}^{*}$, where $F_{n}$ denotes the well known Fourier matrix [31, p. 32].

Lemma 2: For every $q \in\{1,2, \ldots, n-1\}, z \in \mathbb{C}^{n}$ satisfies $\omega^{q} z=\Pi_{n} z$ if and only if $z=v_{q} z_{1}$.

Proof: The statement $\omega^{q} z=\Pi_{n} z$ is equivalent to

$$
\begin{aligned}
z_{2} & =\omega^{q} z_{1} \\
z_{3} & =\omega^{q} z_{2}=\omega^{2 q} z_{1} \\
& \vdots \\
z_{n} & =\omega^{(n-1) q} z_{1},
\end{aligned}
$$

with $\omega^{n q} z_{1}=z_{1}$. Equivalently, $z=v_{q} z_{1}$.

\section{Necessary Conditions for Invariance}

Theorem 3: If formation symmetry $C_{m}$ is invariant under the dynamics (2) for every $m$ that divides $n$, then $A$ is a circulant matrix.

Proof: Theorem 3.1.1 of [31] says that an $n \times n$ matrix $A$ is circulant if and only if it commutes with the fundamental permutation matrix, $\Pi_{n}$. Therefore, it suffices to show that $\Pi_{n} A-A \Pi_{n}=0$.

If formation symmetry $C_{m}$ is invariant for every $m$ that divides $n$ it must be that, in particular, initially symmetric formations satisfying (11) are symmetric for all $t \geq 0$, after Lemma 1. Let $q \in\{1,2, \ldots, n-1\}$ be arbitrary and pick an initial formation $z(0)=v_{q} z_{1}(0)$, where $z_{1}(0) \neq 0$. By Lemma $2, z(0)$ satisfies $\omega^{q} z(0)=\Pi_{n} z(0)$. By Lemma 1, $z(0)$ has symmetry $C_{m}$ with $m=n / \operatorname{gcd}(n, q)$. By assumption, the formation $z(t)$ has symmetry $C_{m}$ for all $t \geq 0$. By differentiating the formation constraint $\omega^{q} z(t)=\Pi_{n} z(t)$ with respect to time, one obtains

$$
\begin{aligned}
\omega^{q} A z(t)=\Pi_{n} A z(t) & \stackrel{(11)}{\Longleftrightarrow}\left(\Pi_{n} A-A \Pi_{n}\right) z(t)=0 \\
& \Longleftrightarrow\left(\Pi_{n} A-A \Pi_{n}\right) v_{q} z_{1}(t)=0,
\end{aligned}
$$

for all $t \geq 0$, using Lemma 2 again in the last step. In particular, since $z_{1}(0) \neq 0,\left(\Pi_{n} A-A \Pi_{n}\right) v_{q}=0$.

By Property 1, $A$ has zero row-sums. Thus, $A v_{0}=0$. Also, because $v_{0}$ is an eigenvector of $\Pi_{n}$ with corresponding eigenvector $\lambda=1, \Pi_{n} v_{0}=v_{0}[31$, pp. 72-73]. Therefore, one finds

$$
\left(\Pi_{n} A-A \Pi_{n}\right) v_{0}=\Pi_{n} A v_{0}-A \Pi_{n} v_{0}=-A v_{0}=0 .
$$

Recall that, $\left[\begin{array}{llll}v_{0} & v_{1} & \cdots & v_{n-1}\end{array}\right]=\sqrt{n} F_{n}^{*}$, where $F_{n}$ is the Fourier matrix [31, p.32]. Therefore, we have shown that $\left(\Pi_{n} A-A \Pi_{n}\right) F_{n}^{*}=0$. Since $F_{n}^{*}$ is invertible, $\Pi_{n} A-A \Pi_{n}=$ 0 . Therefore, $A$ is circulant.

The following example highlights the significance of the assumption that not only is symmetry $C_{n}$ invariant, but also all of its subgroups are invariant under the system's dynamics (further to Example 5).

Example 7: Consider $n=6$ agents initially configured such that $\omega z(0)=\Pi_{6} z(0)$. Suppose the graph $\Gamma(A, z(0))$ is coupled in an all-to-all fashion, as in Fig. 6a. Let $\tilde{A}=$ $\operatorname{circ}(-5,1,1,1,1,1)$ and let $A$ be the matrix $\tilde{A}$ but with its second row replaced by $(1 / 2,-4,1 / 2,1 / 2,2,1 / 2)$. For the initial formation $\omega z(0)=\Pi_{6} z(0)$, Fig. 7 a shows how the cyclic group $C_{6}$ is invariant under the dynamics (2), despite the fact that $A$ is not circulant. In Fig. 7a, the dashed lines connect agents $\{1,2,3,4,5,6\}$, in sequence, at regular intervals during the simulation.

However, consider a different initial formation $\omega^{2} z(0)=$ $\Pi_{6} z(0)$, which has symmetry $C_{3}$ (since $\operatorname{gcd}(6,2)=2$, implying that $m=6 / 2=3$ ). $C_{3}$ is a subgroup of $C_{6}$. The associated formation graph is given in Fig. 6b. Formation symmetry $C_{3}$ is not invariant under the dynamics (2), as one can see from the simulation results of Fig. $7 \mathrm{~b}$, where the dashed lines connect agents $\{1,2,3\}$. As time evolves, the initially equilateral formation becomes only isosceles.

\section{GRAPH SYMMETRY AND INVARIANCE}

We have shown that multiagent systems with circulant connectivity have the attractive property that formation symmetry $C_{n}$ and all of its subgroups are invariant under the system's evolution. Moreover, circulant connectivity among the agents is also necessary to obtain this invariance property. Although Theorems 2 and 3 make no mention of graph symmetry, the condition that $A$ is circulant implies the graph is also symmetric. This result is offered in Proposition 1, but a definition and example are helpful first.

Definition 5 (Graph Symmetry): The graph $\Gamma(A, z(t))=$ $\left(\mathcal{V}_{t}, \mathcal{E}_{t}\right)$ is said to have the symmetry group $G$ at time $t$ if it has the property that for every element $g \in G$, if $v(t) \in \mathcal{V}_{t}$, then $g v(t) \in \mathcal{V}_{t}$, and if $e(t) \in \mathcal{E}_{t}$, then $g e(t) \in \mathcal{E}_{t}$. Moreover, the induced maps $v(t) \mapsto g v(t)$ and $e(t) \mapsto g e(t)$ are permutations.

Note that Definition 5 is consistent with the standard notion of symmetry introduced in Section II-B, which is that every element $g \in G$ leaves the set of points in question unchanged. Here, we have only been more explicit about the fact that vertices map only to vertices, and edges to edges. It should also be emphasized that this definition applies equally to both 


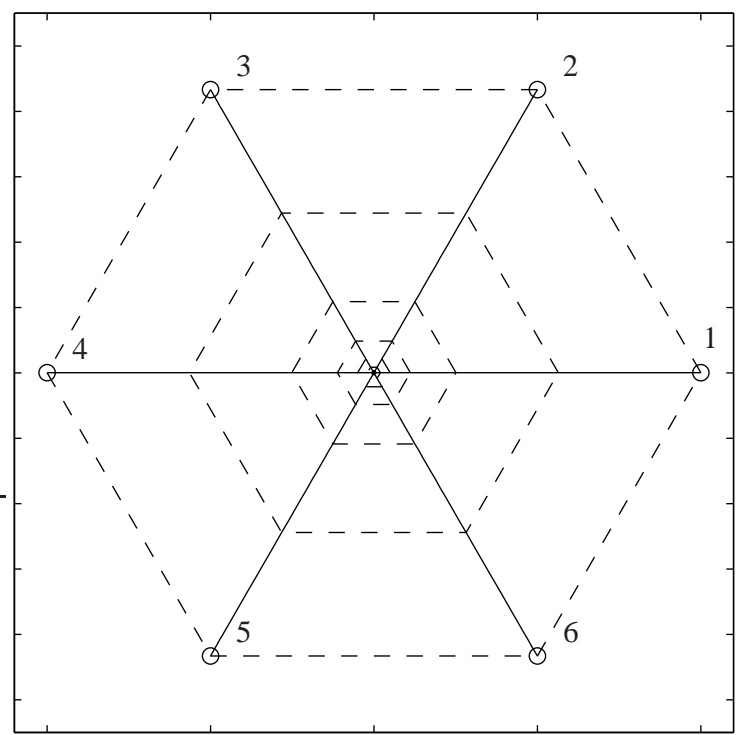

(a) $C_{6}$ symmetry preserved.

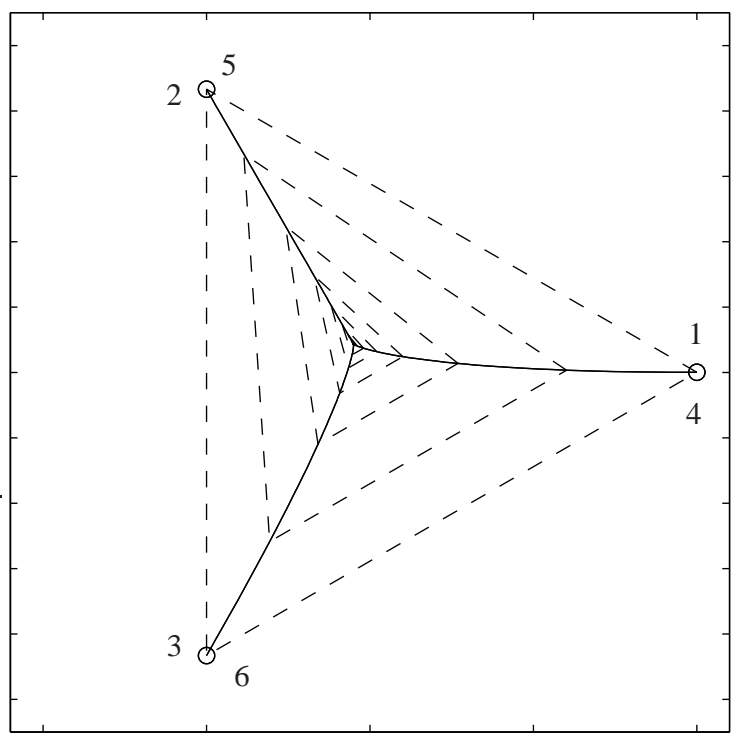

(b) $C_{3}$ symmetry not preserved.

Fig. 7. Simulations for Example 7.

symmetry $C_{m}$ and $D_{m}$. The requirement that the maps in Definition 5 be permutations (hence, bijections) guarantees that two agents (respectively, edges), possibly collocated, cannot be mapped to the same agent (respectively, edge). Notice that graph symmetry $C_{m}$ (Definition 5) implies formation symmetry $C_{m}$ (Definition 3), but not the converse. For instance, the formation $z(t)$ in Fig. $1 \mathrm{~b}$ has formation symmetry $C_{4}$ (the constraint (3) holds with $\sigma=(1,4,2,3)$ ), but the graph $\Gamma(A, z(t))$ has only symmetry $C_{1}$.

Example 8: Consider the graphs $\Gamma(A, z(t))$ of Fig. 1. Fig. 1a has symmetry $C_{2}$, but not $C_{4}$ because a rotation through $\pi / 2$ does not map vertices to vertices. Similarly, Fig. $1 \mathrm{~b}$ has the dihedral group $D_{1}$ but not $D_{2}$ because a reflection about the line through the center with angle $3 \pi / 4$ reverses the edge directions. Fig. 1c has symmetry $C_{1}$, but not $C_{2}$ because a rotation through $\pi$ changes the edge directions.

Proposition 1: Suppose $z(t)$ has symmetry $C_{m}$ with $P_{\sigma}=$ $\Pi_{n}^{q}$. If $A$ is a structurally circulant matrix, then the graph $\Gamma(A, z(t))$ has symmetry $C_{m}$.

Proof: As per Definition 5, it is enough to show that the map induced by a generator of the cyclic group $C_{m}$ maps vertices in $\mathcal{V}_{t}$ (respectively, edge vectors in $\mathcal{E}_{t}$ ) to vertices in $\mathcal{V}_{t}$ (respectively, edge vectors in $\mathcal{E}_{t}$ ) by a bijection. Rotation through $2 \pi / m$ is a generator of the cyclic group $C_{m}$ (cf. Definition 1). Constraint (3) implies the map $z(t) \mapsto e^{j 2 \pi / m} z(t)$ is a bijection on $\mathcal{V}_{t}$, which means that vertices $z_{i}(t) \in \mathcal{V}_{t}$ are mapped to vertices in $\mathcal{V}_{t}$ by a bijection. Consider the rotation of an arbitrary edge vector $e_{i k}(t) \in \mathcal{E}_{t}$ through angle $2 \pi / m$, yielding

$$
\begin{aligned}
e^{j 2 \pi / m} e_{i k}(t) & =e^{j 2 \pi / m}\left(z_{k}(t)-z_{i}(t)\right) \\
& =z_{k+q}(t)-z_{i+q}(t)=e_{i+q, k+q}(t) .
\end{aligned}
$$

Since $e_{i k} \in \mathcal{E}, a_{i k} \neq 0$. But, since $A$ is structurally circulant, $a_{i+q, k+q} \neq 0$, implying that $e_{i+q, k+q} \in \mathcal{E}$. Hence, by the constraint (3), edge vectors $e_{i k}(t) \in \mathcal{E}_{t}$ are mapped to edge vectors in $\mathcal{E}_{t}$ by a bijection ${ }^{2}$.

Example 9: Fig. 4 gives two more example graphs, each with a (structurally) circulant interconnection topology between agents. In each case, one can compare formation symmetry with graph symmetry. Both the formation and graph in Fig. 4a have symmetry $C_{4}$. In this case, the associated permutation is $\sigma(i)=i+1$, which is primitive. In the case of Fig. $4 \mathrm{~b}$, the formation $z(t)$ has symmetry $C_{4}$, but the graph has only symmetry $C_{2}$. In this case, the permutation associated with $C_{2}$ symmetry is $\sigma(i)=i+4$ and $\sigma$ can be factored into exactly four distinct cycles $\sigma=(1,5)(2,6)(3,7)(4,8)$.

The following important example illustrates the fact that graph symmetry is not sufficient to preserve cyclic group symmetries. It also highlights, once again, the importance of the canonical agent labeling described in Section IV-B.

Example 10: Consider a system (2) of $n=4$ agents with $A=\operatorname{circ}(-2,1,2,-1)$. The information flow between agents together with their locations at time $t=0$ is illustrated by $\Gamma(A, z(0))$ in Fig. 4a. Notice that $\Gamma(A, z(0))$ has symmetry $C_{4}$. Clearly, (3) is satisfied with $P_{\sigma}=\Pi_{n}$. Following Theorem 2, this formation's symmetry is invariant under the dynamics (2). But, consider a new initial formation, given by a permutation of the original one, $\tilde{z}(0)=P_{\tau} z(0)$, where $P_{\tau}$ is given by (7). Note that this is a repositioning, not a relabeling (cf. Remark 5). Since the coupling is all-to-all, the new graph $\Gamma(A, \tilde{z}(0))$ still has symmetry $C_{4}$ (any permutation of the agent locations leaves the graph unchanged). However, (3) does not hold with $P_{\sigma}=\Pi_{n}^{q}$ for any $q$, since

$$
P_{\tau} \Pi_{n} P_{\tau}^{\top}=\left[\begin{array}{cccc}
0 & 0 & 1 & 0 \\
0 & 0 & 0 & 1 \\
0 & 1 & 0 & 0 \\
1 & 0 & 0 & 0
\end{array}\right]
$$

${ }^{2}$ Let $\sigma: \mathcal{N} \rightarrow \mathcal{N}$ be a bijection and define $\tau: \mathcal{N} \times \mathcal{N} \rightarrow \mathcal{N} \times \mathcal{N}$ such that $\tau(i, j) \mapsto(\sigma(i), \sigma(j)), i, j \in \mathcal{N}$. Then, $\tau$ is also a bijection. 
is not of the form $\Pi_{n}^{q}$. It can be shown by simulation that symmetry $C_{4}$ in the formation starting at $\tilde{z}(0)$ is not invariant under the dynamics $\dot{\tilde{z}}(t)=A \tilde{z}(t)$, despite the fact that $\Gamma(A, \tilde{z}(0))$ has symmetry $C_{4}$.

\section{DIHEDRAL GROUP FORMATIONS}

By building on the work presented thus far, this last section of the paper considers the invariance of dihedral group formation symmetries. Let $\overline{z(t)}$ denote the complex conjugate of $z(t)$.

Definition 6 ( $D_{m}$ Formation Symmetry): The multiagent formation $z(t) \in \mathbb{C}^{n}$ at time $t$ is said to have symmetry $D_{m}$ if there exist $m$ lines of symmetry

$$
\mathcal{L}_{k}=\left\{z \in \mathbb{C}: z=r e^{j \theta_{k}}, r \in \mathbb{R}\right\} \subset \mathbb{C}, k=1,2, \ldots, m,
$$

with $\theta_{k+1}=\theta_{k}+\pi / m$, and $m$ permutations $\sigma_{k}: \mathcal{N} \rightarrow \mathcal{N}$ such that

$$
e^{j 2 \theta_{k}} \overline{z(t)}=P_{\sigma_{k}} z(t), k=1,2, \ldots, m .
$$

Fig. 8 helps to illustrate how these constraints relate to reflection about a given line passing through the origin in the complex plane. If this definition of $D_{m}$ formation symmetry is to be consistent with the fact that $C_{m} \subset D_{m}$ (see Section II-B), it must be that every $z(t)$ satisfying (14) also satisfies (3).

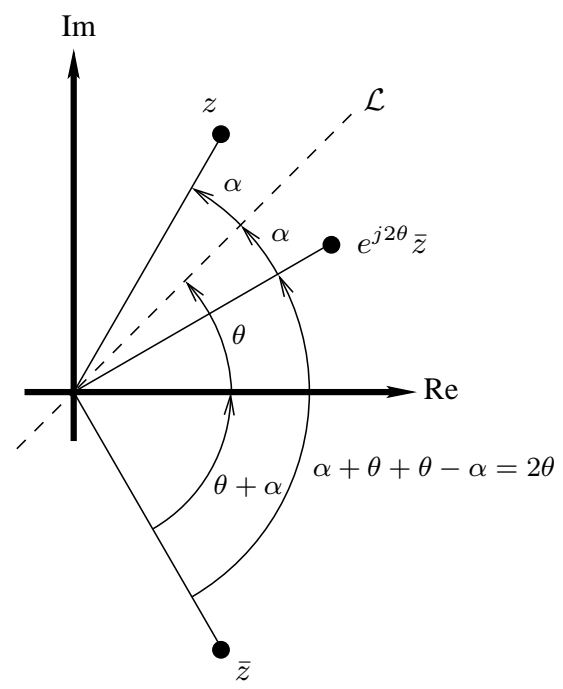

Fig. 8. The reflection of $z \in \mathbb{C}$ about $\mathcal{L} \subset \mathbb{C}$.

Lemma 3: If $z(t) \in \mathbb{C}^{n}$ satisfies (14), then $z(t)$ also satisfies (3).

Proof: It is well known the product of two reflections yields a rotation $\left[29\right.$, p. 35]. Let $\sigma_{k}$ be the permutation of agent indices that corresponds to reflection about $\mathcal{L}_{k}$, where $k \in\{1,2, \ldots, m\}$. Then $\sigma_{k}$ and the formation $z(t)$ satisfy (14). Now, if we reflect $z(t)$ about $\mathcal{L}_{k}$ followed by $\mathcal{L}_{k+1}$ we obtain

$$
e^{j 2 \theta_{k+1}} \overline{e^{j 2 \theta_{k}} \overline{z(t)}}=e^{j 2\left(\theta_{k+1}-\theta_{k}\right)} z(t)=P_{\sigma_{k+1}} P_{\sigma_{k}} z(t) .
$$

Let $P_{\sigma}:=P_{\sigma_{k+1}} P_{\sigma_{k}}$. Since $\theta_{k+1}-\theta_{k}=\pi / m$, the desired constraint (3) is obtained.

\section{A. Counterexample}

We have already seen that circulant connectivity is sufficient to preserve formation symmetry $C_{m}$ for every $m$ that divides $n$. Therefore, does a similar result hold for dihedral group symmetries? The following counterexample illustrates that the answer is, in general, no.

Example 11: Consider two squares of different side-lengths centred at the origin (hence, $n=8$ ), as shown at $t=0$ in Fig. 3b. This initial formation $z(0)$ has symmetry $D_{4}$. Let $A=\operatorname{circ}(-1,-1,0,0,0,0,2,0)$. Simulation results demonstrate that symmetry $C_{4}$ is preserved (i.e., the squares remain squares), but that the two squares (shown as dashed versus dotted lines, at regular intervals, in Fig. 3b) rotate at different rates. Hence, the formation symmetry $D_{4}$ present at $t=0$ is broken.

\section{B. Dihedral Invariance}

Despite the aforementioned counterexample, a circulant system matrix does preserve dihedral group symmetries for the special class of formations introduced in Section V-B. The following lemma extends Lemma 1 to symmetry $D_{m}$.

Lemma 4: Suppose $\omega^{q} z(t)=\Pi_{n} z(t)$ holds for some $q \in$ $\{1,2, \ldots, n-1\}$ and $z(t) \in \mathbb{C}^{n}$. Then, the formation $z(t)$ has symmetry $D_{m}$, where $m=n / \operatorname{gcd}(n, q)$.

Proof: Following Definition 6, we need to show that there exist $m$ lines of symmetry and $m$ corresponding permutations that satisfy (14). The proof is by construction. Pick $\theta_{1}:=$ $\operatorname{Arg}\left(z_{1}(t)\right)$, which is the principal argument of $z_{1}(t) \in \mathbb{C}$. Therefore, $\theta_{k+1}=\theta_{k}+\pi / m, k=1,2, \ldots, m-1$.

First, we show that (14) holds when $k=1$ for some permutation $\sigma_{1}: \mathcal{N} \rightarrow \mathcal{N}$. By applying the left-hand side of (14) to $z_{1}(t)$, one obtains

$$
e^{j 2 \theta_{1}} \overline{z_{1}(t)}=z_{1}(t) .
$$

By doing the same for $z_{i}(t)$, where $i \in \mathcal{N}$ is arbitrary, one obtains

$$
\begin{gathered}
e^{j 2 \theta_{1}} \overline{z_{i}(t)}=e^{j 2 \theta_{1}} \overline{\omega^{(i-1) q} z_{1}(t)}=e^{j 2 \theta_{1}} \omega^{(1-i) q} \overline{z_{1}(t)} \\
\stackrel{(16)}{=} \quad \omega^{(1-i) q} z_{1}(t)=z_{n-i+2}(t) .
\end{gathered}
$$

Therefore, the permutation associated with reflection about $\mathcal{L}_{1}$ is $\sigma_{1}(i)=n-i+2, i=1,2, \ldots, n$.

By definition $\theta_{k}=\theta_{1}+\pi(k-1) / m, k=1,2, \ldots, m$. Therefore, by applying the left-hand side of (14) to $z_{1}(t)$, one obtains

$$
e^{j 2 \theta_{k}} \overline{z_{1}(t)}=e^{j 2 \theta_{1}} e^{j 2 \pi(k-1) / m} \overline{z_{1}(t)} \stackrel{(16)}{=} e^{j 2 \pi(k-1) / m} z_{1} .
$$

But, from the proof of Lemma 1 we know that formations satisfying $\omega^{q} z(t)=\Pi_{n} z(t)$ also satisfy $e^{j 2 \pi / m} z(t)=\Pi_{n}^{l_{q}} z(t)$, where $l_{q}$ satisfies $1=l_{q} k_{q}+l_{m} m, k_{q}=q / \operatorname{gcd}(n, q)$, $l_{q}, l_{m} \in \mathbb{Z}$. This implies that (18) is equivalent to

$$
e^{j 2 \theta_{k}} \overline{z_{1}(t)}=e^{j 2 \pi(k-1) / m} z_{1}(t)=z_{1+l_{q}(k-1)}(t) .
$$

Hence, doing the same for $z_{i}(t)$, where $i \in \mathcal{N}$ is arbitrary, yields

$$
\begin{aligned}
e^{j 2 \theta_{k}} \overline{z_{i}(t)} & =e^{j 2 \theta_{1}} e^{j 2 \pi(k-1) / m} \overline{z_{i}(t)} \\
& \stackrel{(17)}{=} e^{j 2 \pi(k-1) / m} z_{n-i+2}(t)=z_{n-i+2+l_{q}(k-1)}(t) .
\end{aligned}
$$


Therefore, the permutation associated with a reflection about $\mathcal{L}_{k}$ is $\sigma_{k}(i)=n-i+2+l_{q}(k-1), i=1,2, \ldots, n, k=$ $1,2, \ldots, m$, concluding the proof.

Therefore, Lemma 4 and Lemma 3 together yield the following corollary to our principal sufficiency theorem, Theorem 2.

Corollary 2: Suppose $A$ is a circulant matrix and the formation $z(0) \in \mathbb{C}^{n}$ has symmetry $D_{m}$ with $\omega^{q} z(0)=\Pi_{n} z(0)$ for some $q \in\{1,2, \ldots, n-1\}$ satisfying $\operatorname{gcd}(n, q)=n / m$. Then $z(t)$ has symmetry $D_{m}$ with $\omega^{q} z(t)=\Pi_{n} z(t)$ for all $t \geq 0$.

Proof: Since $z(0)$ has symmetry $D_{m}$ at $t=0$ with $\omega^{q} z(0)=\Pi_{n} z(0)$, by definition $z(0)$ has symmetry $C_{m}$ with $P_{\sigma}=\Pi_{n}^{l_{q}}$ as in (13) in the proof of Lemma 1. By Theorem 2, $z(t)$ has symmetry $C_{m}$ with $P_{\sigma}=\Pi_{n}^{l_{q}}$; equivalently $\omega^{q} z(t)=\Pi_{n} z(t)$, for all $t \geq 0$. However, by Lemma 4 , this implies that $z(t)$ has symmetry $D_{m}$ with $\omega^{q} z(t)=\Pi_{n} z(t)$ for all $t \geq 0$, concluding the proof.

\section{CONCLUSION}

By combining the sufficiency result of Theorem 2 and the necessity result of Theorem 3, we have shown that for a multiagent system of the form (1)-(2), formation symmetry $C_{m}$ is invariant under the system's dynamics for every $m$ that divides $n$ if and only if the system has circulant connectivity. Moreover, we have observed that although graph symmetry is not sufficient, it does play a necessary role in symmetry invariance. Finally, dihedral group formations are generally not preserved under circulant connectivity.

A few open questions exist, in light of our results. Firstly, one might naturally wonder about the necessity of the canonical labeling introduced in Sec. IV-B and assumed in Definition 3. Is this labeling assumption without loss of generality? Do there exist other classes of labeling for which there is symmetry invariance if and only if the system matrix is circulant? Secondly, to what extent are the presented results specific to the simple integrator model (1)-(2)? And finally, multiagent systems design is often presented as the problem of synthesizing local control strategies that generate desired global behaviors. Instead, the contributions of this paper emphasize the importance of structure. It seems reasonable that structure could be exploited towards design. Given a set of fixed agent behaviors, can we control a multiagent system's function (e.g., its steady-state and transient behaviors) by intelligently switching the agent interconnection topology? At present, these questions remain as future work.

\section{ACKNOWLEDGMENTS}

The authors would like to thank Bruce Francis and Manfredi Maggiore for their helpful input, and Nicola Ceccarelli for originally pointing out Example 5.

\section{REFERENCES}

[1] S. H. Strogatz, SYNC: How Order Emerges from Chaos in the Universe, Nature, and Daily Life. New York: Hyperion, 2003.

[2] S. Martínez and F. Bullo, "Optimal sensor placement and motion coordination for target tracking," Automatica, vol. 42, no. 4, pp. 661$668,2006$.
[3] J. Cortes, S. Martinez, T. Karratas, and F. Bullo, "Coverage control for mobile sensing networks," IEEE Transactions on Robotics and Automation, vol. 20, no. 2, pp. 243-255, December 2004.

[4] J. Cortes and F. Bullo, "Coordination and geometric optimization via distributed dynamical systems," SIAM Journal on Computing, vol. 44, no. 5, pp. 1543-1574, 2005.

[5] B. Moore and K. Passino, "Distributed balancing of aav's for uniform surveillance coverage," in IEEE Conference on Decision and Control, December 2005

[6] P. Ögren, E. Fiorelli, and N. E. Leonard, "Cooperative control of mobile sensor networks: Adaptive gradient climbing in a distributed environment," IEEE Transactions on Automatic Control, vol. 49, no. 8, pp. 1292-1302, August 2004

[7] L. C. Godara, "Applications of antenna arrays to mobile communications. part i: Performance improvement, feasibility, and system considerations," Proceedings of the IEEE, 1997.

[8] P. Freeman, R. Yang, and K. Lynch, "Distributed estimation and control of swarm formation statistics," in American Control Conference, 2006.

[9] D. Goldenberg, J. Lin, S. Morse, B. Rosen, and R. Yang, "Towards mobility as a network control primitive," in 5th ACM International Symposium on Mobile and Adhoc Networking and Computing, Tokyo, Japan, 2004, pp. 163-174.

[10] E. W. Justh and P. S. Krishnaprasad, "Steering laws and continuum models for planar formations," in Proceedings of the 42nd IEEE Conference on Decision and Control, Maui, Hawaii, December 2003, pp. 3609-3614.

[11] R. Sepulchre, D. Paley, and N. Leonard, "Collective motion and oscillator synchronization," in Cooperative Control: A Post-workshop Volume: 2003 Block Island Workshop on Cooperative Control, ser. Lecture Notes in Control and Information Sciences, V. J. Kumar, N. E. Leonard, and A. S. Morse, Eds., vol. 309. Spring-Verlag, Inc., 2004, pp. 189-205.

[12] I. Suzuki and M. Yamashita, "Distributed anonymous mobile robots: Formation of geometric patterns," SIAM Journal of Computing, vol. 28, no. 4, pp. 1347-1363, 1999.

[13] K. Sugihara and I. Suzuki, "Distributed motion coordination of multiple mobile robots," in Proceedings of the 5th IEEE International Symposium on Intelligent Control, 1990, pp. 138-143.

[14] N. E. Leonard and E. Fiorelli, "Virtual leaders, artificial potentials and coordinated control of groups," in Proceedings of the 40th IEEE Conference on Decision and Control, Orlando, Florida, 2001, pp. 29682973.

[15] M. Egerstedt and X. Hu, "Formation constrained multi-agent control," IEEE Transactions on Robotics and Automation, vol. 17, no. 6, pp. 947951, December 2001.

[16] R. Fierro, A. K. Das, V. Kumar, and J. P. Ostrowski, "Hybrid control of formations of robots," in Proceedings of IEEE International Conference on Robotics and Automation, Seoul, Korea, May 2001, pp. 157-162.

[17] P. Krishnaprasad, "Relative equilibria and stability of rings of satellites," in IEEE Conference on Decision and Control, vol. 2, December 2000, pp. $1285-1288$.

[18] H. Yamaguchi and J. W. Burdick, "Asymptotic stabilization of multiple nonholonomic mobile robots forming group formations," in IEEE International Conference on Robotics and Automation, 1998, pp. 3573-3580.

[19] H. Yamaguchi, "A distributed motion coordination strategy for multiple nonholonomic mobile robots in cooperative hunting operations," Robotics and Autonomous Systems, vol. 43, no. 4, pp. 257-282, 2003.

[20] H. Yamaguchi and J. W. Burdick, "Time-varying feedback control for nonholonomic mobile robots forming group formations," in IEEE Conference on Decision and Control, Tampa, Florida, USA, December 1998, pp. 4156-4163.

[21] J. A. Fax and R. M. Murray, "Information flow and cooperative control of vehicle formations," IEEE Transactions on Automatic Control, vol. 49, no. 9, pp. 1465-1476, September 2004.

[22] A. Pogromsky, G. Santoboni, and H. Nijmeijer, "Partial synchronization: From symmetry towards stability," Physica D, vol. 172, pp. 65-87, 2002.

[23] B. Recht and R. D'Andrea, "Distributed control of systems over discrete groups," IEEE Transactions on Automatic Control, vol. 49, no. 9, pp. 1446-1452, September 2004.

[24] A. M. Bruckstein, G. Sapiro, and D. Shaked, "Evolutions of planar polygons," International Journal of Pattern Recognition and Artificial Intelligence, vol. 9, no. 6, pp. 991-1014, 1995.

[25] T. J. Richardson, "Stable polygons of cyclic pursuit," Annals of Mathematics and Artificial Intelligence, vol. 31, pp. 147-172, 2001.

[26] B. Nabet and N. Leonard, "Shape control of a multi-agent system using tensegrity structures," in 3rd IFAC Workshop on Lagrangian and Hamiltonian Methods for Nonlinear Control, July 2006. 
[27] F. Zhang, M. Goldgeier, and P. Krishnaprasad, "Control of small formations using shape coordinates," in IEEE International Conference on Robotics and Automation, vol. 2, June 2003, pp. 1074-1079.

[28] H. Yamaguchi and T. Arai, "Distributed and autonomous control method for generating shape of multiple mobile robot group," in IEEE/RSJ International Conference on Intelligent Robots and Systems, Munich, Germany, September 1994, pp. 800-807.

[29] H. S. M. Coxeter, Regular Polytopes. London: Methuen \& Co. Ltd., 1948.

[30] — Introduction to Geometry, 2nd ed., ser. Wiley Classics Library. Toronto: John Wiley \& Sons, Inc., 1989.

[31] P. J. Davis, Circulant Matrices, 2nd ed. New York: Chelsea Publishing, 1994.

[32] H. S. M. Coxeter and W. O. J. Moser, Generators and Relations for Discrete Groups, 4th ed. Berlin: Springer-Verlag, 1980.

[33] H. S. M. Coxeter, Regular Complex Polytopes, 2nd ed. Cambridge: Cambridge University Press, 1991.

[34] J. Bang-Jensen and G. Gutin, Digraphs: Theory, Algorithms and Applications. Springer-Verlag, 2002.

[35] C.-T. Lin, "Structural controllability," IEEE Transactions on Automatic Control, vol. AC-19, no. 3, pp. 201-208, June 1974.

[36] A. Bernhart, "Polygons of pursuit," Scripta Mathematica, vol. 24, pp. 23-50, 1959.

[37] J. A. Marshall, M. E. Broucke, and B. A. Francis, "Formations of vehicles in cyclic pursuit," IEEE Transactions on Automatic Control, vol. 49, no. 11, pp. 1963-1974, November 2004.

[38] G. A. Jones and J. M. Jones, Elementary Number Theory, ser. Springer Undergraduate Mathematics Series. London: Springer-Verlag, 1998. 